\title{
Embarazo ectópico y abdominal, Hospital de Caldas 1970-1993
}

\author{
Eduardo León Jaramillo V.*; Jhon Jairo Castro J.**; Rafael Angel G.***
}

\begin{abstract}
RESUMEN: Se informa de una paciente con embarazo abdominal de 24 semanas de gestación, atendida en el Hospital de Caldas, Manizales. Se analiza y se compara con otras regiones la incidencia de embarazo abdominal y ectópico y las muertes maternas relacionadas con estas causas, durante el período 1970-1993.
\end{abstract}

\section{PALABRAS CLAVES: Embarazo, Ectópico, Abdominal}

SUMMARY: A patient with a 24 weeks abdominal pregnancy who was attended in Hospital de Caldas, Manizales, Colombia, is reported. The report presents data regarding the number and rate of ectopic and abdominal pregnancies and pregnancy-related deaths in Hospital de Caldas, from 1970 through 1993.

KEY WORDS: Pregnancy, Ectopic, Abdominal

\section{Caso clínico}

El 7 de octubre de 1993 ingresó por urgencias del Hospital de Caldas, Manizales, Colombia, remitida de un puesto de salud rural, paciente de 26 años G2P1A0, con 22 semanas de embarazo según amenorrea confiable, quien consultó por presentar dolor abdominal de 4 semanas de evolución. Durante la última semana el dolor se intensificó y, además, empezó a presentar equimosis en hemiabdomen inferior.

$\mathrm{Al}$ examen físico se encontró palidez intensa de piel y mucosas, FC 105/min., FR 22/min., abdomen con signo de Cullen, blando, depresible, peristaltismo positivo, útero gravido, AU $35 \mathrm{~cm}$., no se encontró frecuencia cardíaca fetal. Se realizó ecografía que mostró feto vivo con edad gestacional coincidente con FUM y presencia de ascitis. Por paracentesis se obtuvo sangre no coagulable.

Fue hospitalizada con los siguientes diagnósticos: embarazo de $+\backslash-22$ semanas, úlcera péptica perforada y pancreatitis.

El laboratorio clínico informó hemoglobina $4.4 \mathrm{gr} \%$, leucocitos 21,900 , neutrófilos $90 \%$, linfocitos $10 \%$, recuento de plaquetas 375,000 , VSG 66, TP 13.2" (control 12 ”), TPT 28.5" (control 30.6"), Grupo B Rh positivo.

Mediante laparotomía exploratoria se encontró embarazo abdominal de +/- 24 semanas, con feto vivo, placenta adherida a epiplón mayor, cuerno uterino derecho, trompa derecha, ovario derecho, colon sigmoide y recto; hemoperitoneo de $+/-3,500 \mathrm{cc}$; destrucción total

\footnotetext{
* MD Epidemiólogo Residente I de Ginecología y Obstetricia.

** MD Ginecoobstetra Hospital de Caldas.

*** MD Cirujano Hospital de Caldas.
}

de todo el cuerno, trompa y ovario derechos; se resecó parte de placenta la cual estaba necrótica y parte se dejó in situ; hemorragia abundante de lecho placentario; con gran dificultad se hizo hemostasia.

Se le transfundieron 10 unidades de sangre fresca total. La paciente evolucionó tórpidamente, desarrolló sepsis, falla multisistémica y falleció.

Anatomía patológica informó "trombosis vascular, miometritis, necrosis de placenta, corioamnionitis aguda", de útero tomado postmortem.

\section{Incidencia}

Motivados por este caso, revisamos la información disponible en la Sección de Estadística del Hospital de Caldas encontrando que, durante el período 1970-1993, fueron hospitalizadas 1,325 pacientes por embarazo ectópico y ocurrieron 94, 332 nacimientos, para una tasa media de 14.1 embarazos ectópicos por 1,000 nacimientos (tabla 1), con cifras fluctuantes de incidencia anual que van desde 6.6 por 1,000 en 1978 hasta 23.5 por 1,000 en 1993, y con tendencia significativa al aumento ( $p$ menor que 0.05), (Figura 1).

Uno (1) de los casos ocurrido en 1975 fue abdominal, con sobrevivencia de la paciente. Este caso, sumado al que estamos describiendo en este artículo, nos da una tasa de 2.1 embarazos abdominales por 100,000 nacimientos y 1.5 embarazos abdominales por 1,000 ectópicos, para el período descrito.

Tres (3) de las pacientes fallecieron por complicaciones del ectópico para una tasa de letalidad media de 22.6 por 10,000 ectópicos y tasas de $23.3,13.7$ y 12.7 por 10.000 ectópicos, para los años 76, 84 y 93 respectivamente, en que ocurrieron las muertes. 


\section{Tabla 1 \\ NACIMIENTOS, NUMERO Y TASA DE EMBARAZOS ECTOPICOS HOSPITAL DE CALDAS, 1970-1993}

\begin{tabular}{|c|c|c|c|}
\hline Año & $\begin{array}{l}\text { Nacidos } \\
\text { Vivos }\end{array}$ & $\begin{array}{l}\text { Embarazos } \\
\text { Ectópicos }\end{array}$ & $\begin{array}{l}\text { Tasa } \\
\text { Por mil } \\
\text { N. V. }\end{array}$ \\
\hline 1970 & 2,607 & 44 & 16.9 \\
\hline 1971 & 3,254 & 35 & 10.8 \\
\hline 1972 & 2,869 & 54 & 18.8 \\
\hline 1973 & 2,923 & 29 & 9.9 \\
\hline 1974 & 3,009 & 37 & 12.3 \\
\hline 1975 & 3,067 & 38 & 12.4 \\
\hline 1976 & 3,217 & 43 & 13.4 \\
\hline 1977 & 3,466 & 37 & 10.7 \\
\hline 1978 & 3,649 & 24 & 6.6 \\
\hline 1979 & 3,893 & 33 & 8.5 \\
\hline 1980 & 4,143 & 52 & 12.6 \\
\hline 1981 & 4,411 & 75 & 17.0 \\
\hline 1982 & 4,702 & 61 & 13.0 \\
\hline 1983 & 4,647 & 49 & 10.5 \\
\hline 1984 & 4,740 & 73 & 15.4 \\
\hline 1985 & 4,672 & 61 & 13.1 \\
\hline 1986 & 4,627 & 48 & 10.4 \\
\hline 1987 & 4,753 & 71 & 14.9 \\
\hline 1988 & 4,561 & 89 & 19.5 \\
\hline 1989 & 4,413 & 63 & 14.3 \\
\hline 1990 & 4,561 & 79 & 17.3 \\
\hline 1991 & 4,766 & 70 & 14.7 \\
\hline 1992 & 4,017 & 81 & 20.2 \\
\hline 1993 & 3,365 & 79 & 23.5 \\
\hline Total & 94,332 & 1,325 & 14.1 \\
\hline
\end{tabular}

\section{Discusión}

No disponemos de datos colombianos sobre incidencia de embarazo abdominal durante el período descrito que nos permitan hacer comparaciones con nuestras cifras y sólo encontramos la publicación reciente de González M. y Heredia H. (1) describiendo un caso presentado en el Hospital de Sogamoso, sin análisis de incidencia en ese hospital.

Jubiz (2) refiere que en la literatura colombiana se encuentran publicados 32 casos de embarazo abdominal hasta el año de 1960. La obstetricia de Williams (3) refiere que ocurren 9.2 embarazos abdominales por cada 1,000 embarazos ectópicos en los Estados Unidos y cita estudios del Charity Hospital de Nueva Orleans (1962) y del Indiana University Hospital (1977), que refieren 1 embarazo abdominal por 3,337 nacimientos y 1 por 7,931 nacimientos, respectivamente.

El Centro de Control de enfermedades de los Estados Unidos estima que entre 1970 y 1989 fueron hospitalizadas 1'047,900 mujeres norteamericanas por embarazo ectópico, para una tasa media de 14.8 por 1,000 nacimientos, con una tendencia ascendente a través del período y un incremento de 4.8 en 1970 a 22.0 en 1989 (4).

Lo que ocurre en los Estados Unidos parece ser un fenómeno mundial y es analizado por Makinen (5) quien explica el aumento de embarazos ectópicos en Finlandia como un efecto de cohortes del baby boom postguerra y del aumento de enfermedades de transmisión sexual que acompañó a la revolución sexual de principios de los años 60 .

Nuestras tasas, tanto de embarazo abdominal como de ectópico, son más bajas comparativamente con las cifras expuestas para hospitales norteamericanos.

Creemos, sin embargo, que tenemos un subregistro considerable y nos lo confirman los obstetras que han ejercido en el hospital desde hace varias décadas y que nos informan de casos de embarazo abdominal que ellos operaron durante el período descrito, que no fueron publicados ni adecuadamente consignados en los registros de la institución. Además, cuando revisamos en la oficina de estadística la información sobre el caso que hemos descrito en este artículo, lo encontramos mal codificado, con el código 633.X correspondiente a embarazo ectópico no especificado, en lugar del 633.0 que le corresponde según la Clasificación Internacional de Enfermedades (6).

Si bien el número de muertes maternas (3) por embarazo ectópico ocurridas en el Hospital de Caldas durante los 24 años de este estudio es bajo, no podemos quedarnos en la cifra absoluta. De hecho, al estimar las tasas de letalidad y compararlas con las de los Estados Unidos para un período similar, nuestras cifras son elevadas (Tasa media $=22.6$ muertes por 10,000 ectópicos). Sin embargo, hemos tenido un descenso del $54.5 \%$ de 23.3 (1976) a 12.7 (1993) muertes maternas por 10,000 ectópicos.

En los Estados Unidos el embarazo ectópico es la principal causa de muerte materna durante el primer trimestre del embarazo, no obstante haber logrado un descenso de 35.5 a 3.5 muertes maternas por 10,000 ectópicos entre 1970 y 1989 , (4).

A diferencia de lo que sucede en los Estados Unidos y en países desarrollados, en países en desarrollo el embarazo ectópico no tiene un peso relativo muy importante en la estructura de la mortalidad materna. El hecho es explicado por el desplazamiento que hacen del ectópico causas como sepsis, toxemia y hemorragia, de muy elevada frecuencia entre nosotros y que explican más del $90 \%$ de muertes maternas en hospitales como el nuestro (7). 
Figura 1

TENDENCIA DE EMBARAZO ECTOPICO

HOSPITAL DE CALDAS, MANIZALES, 1970 - 1993

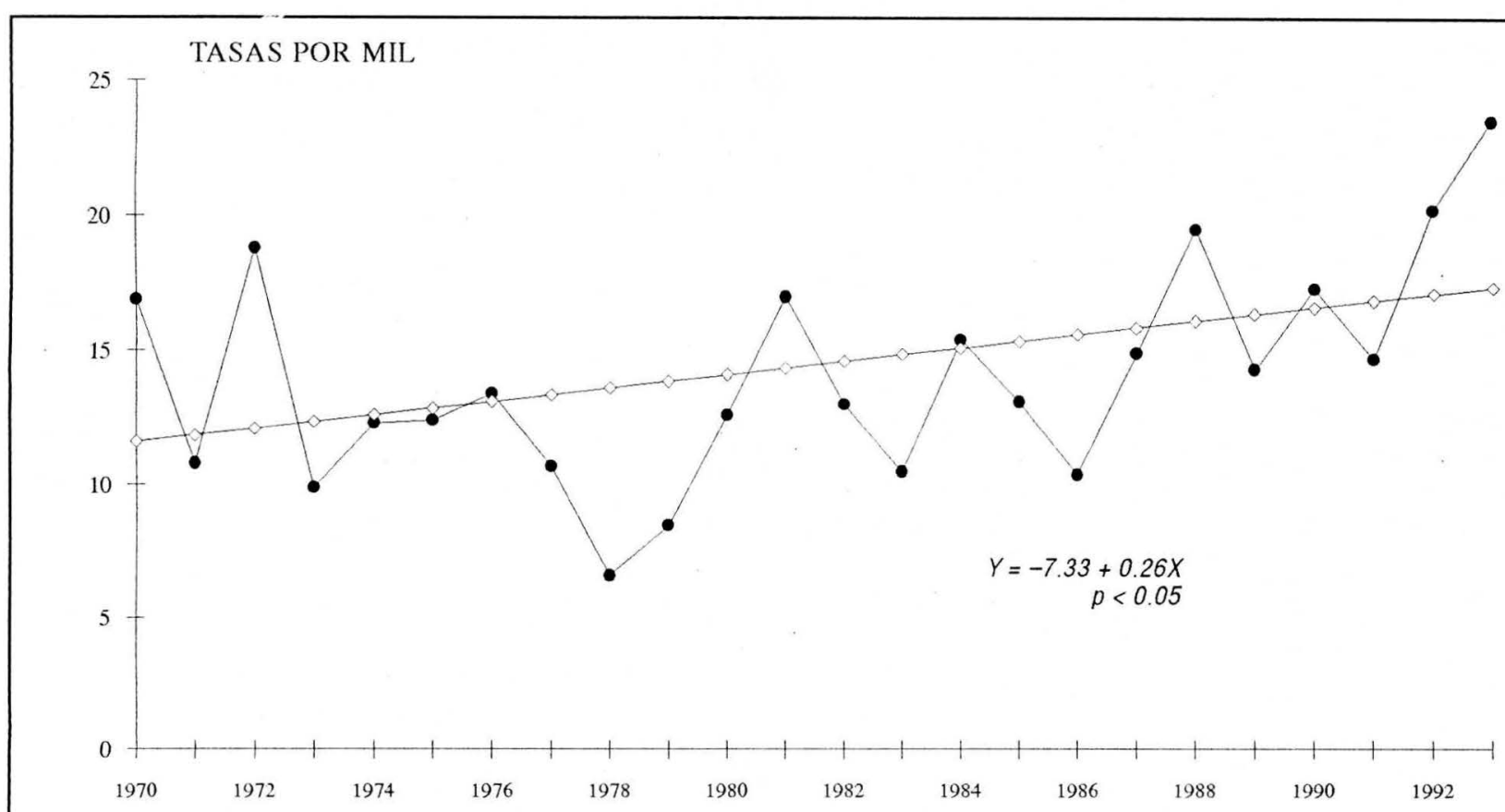

FUENTE: S. DE ESTADISTICA, H. DE CALDAS

No obstante la imperfección de nuestros sistemas de información, las tasas anuales de incidencia de embarazo ectópico presentadas en este artículo, nos muestran un problema creciente de salud pública para el departamento de Caldas.

En consecuencia, debemos diseñar un sistema de vigilancia epidemiológica que nos permita una recolec- ción prospectiva de datos de buena calidad y con ello podamos tanto describir las características de nuestras pacientes con embarazo ectópico como embarcarnos en estudios analíticos que aumenten nuestro conocimiento sobre factores de riesgo asociados con esta causa de morbilidad y mortalidad materna.

\section{BIBLIOGRAFIA}

1. González Mariño MA., Heredia Hernández MM. Embarazo abdominal. Rev. Col. de Obstet. y Gynecol. 1992; 43 (1): 70-71.

2. Jubiz Hasbun A. Embarazo ectópico. In: Botero UJ., Jubiz HA., Henao G. Obstetricia y Ginecología. 1992, 2a. reimp. 4a. ed.

3. Pritchard JA., Macdonald PC., Gant NF Williams. Obstetricia, 3a. ed. Salvat $1987 ; 419-422$.

4. CDC. Ectopic pregnancy - United States, 1988-1989. NMWR, 1992; 41 (32): 591-594.
5. Makinen JI. Increase of ectopic pregnancy in Finland Combination of time and cohort effects. Obstet. and Ginecol. 1989; 73 (1): 21-24.

6. OPS/OMS. Clasificación internacional de enfermedades. 9a. revisión, 1975

7. Jaramillo Velásquez EL., El Kouri de LN., Villa ML. Mortalidad Materna en el Hospital San Jorge. Pereira 1972-1986. Bol. epidemiol. del Serv. Secc. de Salud de Ris. No. 1, 1987. 